\section{Breast cancer therapy with IL-25}

\section{By Michael J. Haas, Senior Writer}

A team of U.S. and Taiwan researchers has shown that IL-25, a proinflammatory cytokine involved in allergic inflammation, also exerts potent cytotoxic effects in breast cancer cells and decreases mammary tumor growth in mice. ${ }^{1}$ The researchers are licensing the findings to an undisclosed company, and the partners will develop systems for delivering IL-25 or its mimetics directly to tumors.

In 2005, researchers at the University of California, Irvine School of Medicine reported in the Proceedings of the National Academy of Sciences that the medium in which normal human mammary epithelial cells had been cultured exerted cytotoxic effects on human breast cancer cell lines. ${ }^{2}$

The U.S.-Taiwan team-which included two researchers from the PNAS study-now has identified IL-25 (IL-17E) as the most potent cytotoxic component in the medium.

The team then isolated IL-25 from normal human epithelial cells and showed that the cytokine bound its receptor, IL-17 receptor $B$ (IL-17RB; IL-25R), on multiple breast cancer cell lines and increased apoptosis compared with vehicle.

In mice with xenograft mammary tumors, injections of IL-25 lowered tumor growth compared with injections of vehicle.

Paradoxically, IL-25R was highly expressed on multiple human breast cancer cell lines compared with normal breast cell lines, and IL-25R expression in primary breast tumors correlated with poor prognosis and high mortality. One possible explanation, the team wrote in its report in Science Translational Medicine, is that IL-25 is absent in breast tumors and something else is binding the receptor to promote a tumorigenic rather than an apoptotic response.

The likely culprit, they wrote, "appears to be IL-17B," which is another IL-25R ligand that shares less than 50\% homology with IL-25.

Additional cell line experiments showed that IL-25 activated apoptosis by binding to a death domain-like region of its receptor. By contrast, IL-17B may bind the receptor in a different manner to promote tumor cell growth, the team wrote.

"It is remarkable that IL-25 has a strong cytotoxic effect that inhibits cancer progression, and the increased level of IL-25R in breast cancer cells is interesting," said Seon Hee Chang, instructor in the Department of Immunology at The University of Texas M.D. Anderson Cancer Center. Chang coauthored a recent review article on IL-17 proteins. $^{3}$

\section{Life and death questions}

Chang said future studies should investigate precisely how and why IL-25 triggers apoptosis in cancer cells.

"No one has previously reported this cytotoxic activity for IL-25," she said. "It might not be related to the death domain on IL-25R" because noncancerous, IL-25R-expressing cells do not undergo apoptosis in response to IL-25-thus suggesting the apoptotic effect might be specific to cancer cells, she said.

Chang also wanted to see studies on the potentially cancer-promoting interactions between IL-17B and IL-25R.

In terms of safety, Chang said, "IL-25 is known to promote allergic inflammation, and it recruits and expands eosinophils and other innate immune cells that produce IL-4, IL-5 and IL-13," which are involved in T helper type 2 cell responses. Thus, she said, future studies also should address the potential for IL-25 therapy to cause inflammatory side effects.

Saori Furuta, first author on both the recent paper and the 2005 PNAS study, noted that the team's experiments in xenograft models already showed that IL-25 may have a favorable safety profile.

At doses threefold to fivefold higher than those used in the efficacy experiments, IL-25 had "no adverse effect on the animals' physiology or the pathology of tissues and organs," she said. "This observation suggests that administration of IL-25 does not induce systemic stress, such as inflammatory responses."

Nevertheless, Furuta said the team plans to collaborate with its corporate partner to develop tumor-targeting systems to deliver IL-25 or IL-25 peptidomimetics directly to breast tumors, thereby decreasing any potential for side effects.

Furuta is a postdoctoral fellow in the group of Mina Bissell, who is a distinguished scientist at Lawrence Berkeley National Laboratory. Bissell and Wen-Hwa Lee, professor of biomedicine at the UCI School of Medicine, co-led the team, which included a researcher from the National Taiwan University Hospital.

The group also is investigating the effects of IL-17B signaling through IL-25R in breast cancer, said Furuta.

She noted that the team's study identified five other components from conditioned medium that had cytostatic-but not cytotoxicactivity in breast cancer cells: anti-thrombin III (AT3; SERPINC1), bone morphogenetic protein 10 (BMP10), fibroblast growth factor-11 (FGF-11), group-specific component (vitamin D binding protein) (GC; VDBP) and IL-1 family member $7 \delta$ (IL-1F7).

"We have been testing whether combining these factors with IL-25 might lead to additive or synergistic effects on tumor cells," she said.

Lawrence Berkeley and UCI have filed for a patent covering the findings, and the team is in the process of licensing that IP to an undisclosed company, Furuta said.

Haas, M.J. SciBX 4(18); doi:10.1038/scibx.2011.502

Published online May 5, 2011

\section{REFERENCES}

1. Furuta, S. et al. Sci. Transl. Med.; published online April 13, 2011; doi:10.1126/scitranslmed.3001374 


\section{ANALYSIS}

Contact: Mina J. Bissell, Lawrence Berkeley National Laboratory, Berkeley, Calif.

e-mail: mjbissell@lbl.gov

Contact: Saori Furuta, same affiliation as above

e-mail: sfuruta@|bl.gov

Contact: Wen-Hwa Lee, University of California, Irvine, Calif.

e-mail: whlee@uci.edu

2. Furuta, S. et al. Proc. Natl. Acad. Sci. USA 102, 9176-9181 (2005)

\section{TARGETS \& MECHANISMS}

3. Chang, S.H. \& Dong, C. Cell. Signal. 23, 1069-1075 (2011)

COMPANIES AND INSTITUTIONS MENTIONED

Lawrence Berkeley National Laboratory, Berkeley, Calif.

National Taiwan University Hospital, Taipei, Taiwan

University of California, Irvine School of Medicine, Irvine, Calif.

The University of Texas M.D. Anderson Cancer Center,

Houston, Texas 